\title{
Measurement of spin-lattice relaxation times with longitudinal detection
}

\section{Journal Article}

\section{Author(s):}

Granwehr, J.; Schweiger, A.

Publication date:

2001

Permanent link:

https://doi.org/10.3929/ethz-b-000422494

Rights / license:

In Copyright - Non-Commercial Use Permitted

Originally published in:

Applied Magnetic Resonance 20(1-2), https://doi.org/10.1007/BF03162315 


\title{
Measurement of Spin-Lattice Relaxation Times with Longitudinal Detection
}

\author{
J. Granwehr and A. Schweiger \\ Physical Chemistry Laboratory, Swiss Federal Institute of Technology, Zurich, Switzerland
}

Received January 9, 2001

\begin{abstract}
New experimental schemes to measure spin-lattice relaxation times $T_{1}$ on the basis of inversion-recovery and saturation-recovery experiments with longitudinal detection are introduced. With this approach, paramagnetic species with $T_{1}$ values as short as 20 ns can be measured. Possibilities to reduce unwanted signals and instrumental artifacts are analyzed. An experiment where the signal is induced directly by the time-dependent $M$. magnetization is also proposed. Experimental results for organic radicals and defect centers are presented and compared with data obtained with conventional techniques, and a metal complex at $250 \mathrm{~K}$ is analyzed where it is very difficult to get information about relaxation times with established methods because of fast spin-spin relaxation.
\end{abstract}

\section{Introduction}

During the last five decades, a variety of different methods have been developed to determine the spin-lattice relaxation time $T_{1}$ of paramagnetic species. Among the most popular techniques are the inversion-recovery (IR) [1] and the saturation-recovery (SR) [2] experiments. The major drawback of these methods which measure transverse magnetization is that either an additional low-power pulse channel is required for probe-pulse detection or the spin-spin relaxation time $T_{2}$ has to be long enough to observe an electron spin echo. As an alternative, $T_{1}$ can be determined with longitudinal detection (LOD), where the change in the longitudinal $M_{z}$ magnetization is recorded with a pickup coil. A variety of LODEPR methods have been proposed, as for example, saturation methods [3], the analysis of the signal induced by the return of $M_{z}$ to Boltzmann equilibrium ([4] and P. Höfer, pers. commun.), techniques where the detection frequency is varied [5], and pulse LOD-EPR schemes [6]. All these methods are deadtime free, but most of them suffer from a low sensitivity and require a dedicated instrumental setup.

Recent developments in instrumentation of pulse LOD-EPR on the basis of a commercially available spectrometer [7] now open new possibilities for the determination of $T_{1}$ with high sensitivity. In this contribution we present pulse LOD 
experiments, where the IR or SR preparation sequence is combined with a new detector sequence, and demonstrate the applicability of the methods for the direct measurement of short $T_{1}$ values (P. Höfer, pers. commun.). The study is focused on the realization and optimization of the experiments and on the data analysis. The potentials and limitations of the approaches are demonstrated for different types of samples. First, the methods are applied to samples such as $\alpha, \alpha-$ diphenyl- $\beta$-picrylhydrazyl (DPPH) and $\gamma$-irradiated herasil glass, where $T_{1}$ can also be obtained with conventional techniques. Second, it is demonstrated that $T_{1}$ times of metal complexes at noncryogenic temperatures can be measured, which is usually not possible with methods using detection of the transverse magnetization because of the short $T_{2}$ times. As an example, the relaxation processes of a natural dolomite, where both $\mathrm{Ca}(\mathrm{II})$ and $\mathrm{Mg}(\mathrm{II})$ sites are substituted by $\mathrm{Mn}$ (II), are studied.

\section{Theory}

\subsection{Inversion- and Saturation-Recovery Experiments}

After excitation, the return of the longitudinal component of the magnetization to thermal equilibrium can often be described by [8]

$$
M_{z}(t)=M_{\mathrm{eq}}+\left[M_{z}(0)-M_{\mathrm{eq}}\right] \exp \left(\frac{-t}{T_{1}}\right),
$$

where $M_{\text {eq }}$ is the magnetization at Boltzmann equilibrium, $M_{z}(0)$ is the longitudinal magnetization at the beginning of the relaxation process, and $M_{z}(t)$ is the value of the $z$-magnetization at time $t$.

In an IR experiment, $M_{\text {eq }}$ is inverted with a $\pi$ preparation pulse, and $M_{z}(t)$ is read out with an appropriate detector sequence, usually a probe pulse or a twopulse echo. In the case of echo detection, time $T$ between inversion and detection is increased step by step, and the recovery is recorded point by point, while with probe-pulse detection the recovery is recorded in one shot, resulting in a shorter measuring time. In an SR experiment, a saturation instead of an inversion pulse is applied at the beginning of the sequence.

The main problem with detection of the transverse magnetization is the dependence of the signal intensity on $T_{2}$. In the case of probe-pulse detection, the signal is proportional to $T_{2}$ if $T_{1} \gg T_{2}$ and $1 / T_{2} \gg \omega_{1}$, where $\omega_{1}$ is the amplitude of the microwave ( $\mathrm{mw}$ ) field in angular frequencies [9]. With the two-pulse echo detection sequence, $T_{2}$ has to be longer than the instrumental deadtime. This is usually the case for transition metal compounds at cryogenic temperatures and for radicals at ambient temperatures. At room temperature, transition metal complexes have very short $T_{2}$ times, which makes it difficult or even impossible to measure $T_{1}$ with standard techniques.

A different approach is to measure directly $M_{z}(T)$. This can be achieved by changing the $M_{z}$ magnetization at time $T$ by a microwave (mw) pulse which in 
turn induces a voltage proportional to $M_{z}(T)$ in a pickup coil oriented along the $\mathbf{B}_{0}$-field direction. The coil can be placed inside the $\mathrm{mw}$ resonator and is part of a serial LCR resonant circuit with resonant frequency $\omega_{\mathrm{LCR}}$ and quality factor $Q_{\mathrm{LCR}}$. The time constant of the detection circuit is given by $\tau_{\mathrm{LCR}}=2 Q_{\mathrm{LCR}} / \omega_{\mathrm{LCR}}$. The relaxation behavior is measured in an analogous way as in an IR or SR experiment with echo detection by increasing time $T$ step by step. The pulse scheme for the inversion-recovery longitudinally detected $T_{1}$ experiment (IR-LOD $T_{1}$ ), the progression of $M_{z}$, and the response of the detection circuit are shown in Fig. 1. The saturation-recovery longitudinally detected $T_{1}$ experiment (SR-LOD $T_{1}$ ) is analogous, with the inversion pulse replaced by a saturation pulse. The signal in Fig. 1c for $t \geq T$ can be detected with a transient recorder. Instead of a transient recorder, a boxcar averager can be used. To avoid a cancellation of the signal by the averaging process of the boxcar, either the length of the boxcar window has to be adjusted to a value $t_{\mathrm{box}}<T_{\mathrm{LCR}} / 2$ with the period $T_{\mathrm{LCR}}=2 \pi / \omega_{\mathrm{LCR}}$ and the window has to be properly positioned, or the signal has to be rectified before data acquisition. With a boxcar averager, the amount of data is by orders of magnitude lower than those obtained with a transient recorder, since for each

a
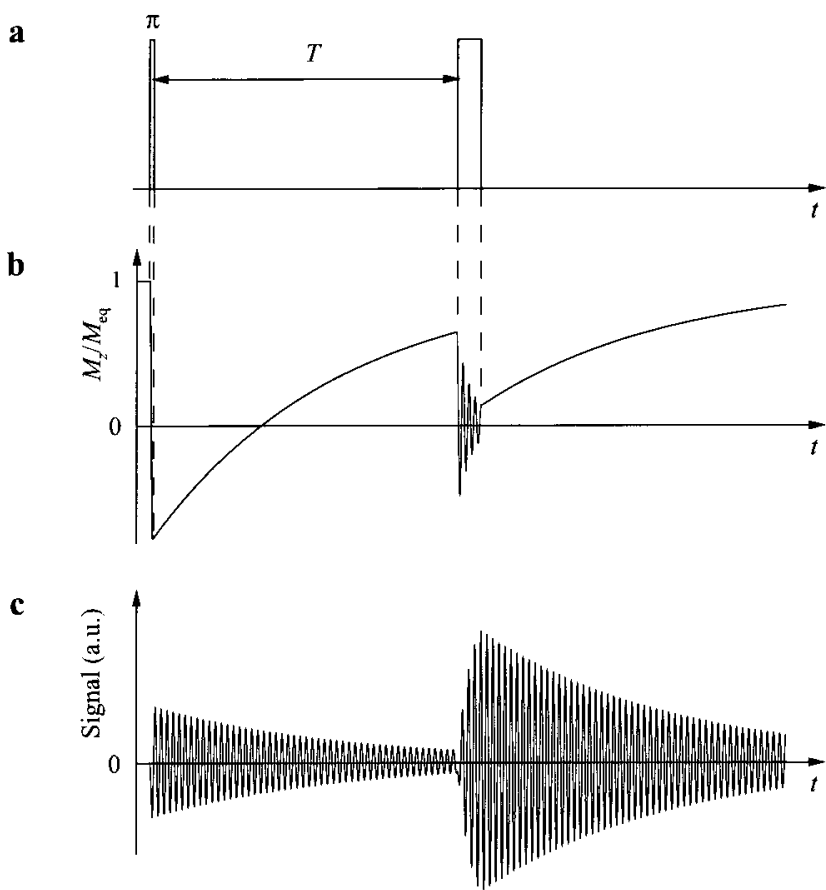

Fig. 1. The IR-LOD $T_{1}$ experiment. a Pulse sequence consisting of an inverting $\pi$ pulse followed by a free evolution period of time $T$ during which the magnetization relaxes towards the Boltzmann equilibrium. At time $T$ a nutation pulse is applied to read out $M_{z}(T)$. b Progression of $M_{z}$ as a function of time $t$. In this simulation a ratio between the longitudinal and the transverse relaxation time of $T_{1} / T_{2}=25$ is used. c Simulated response of a serial LCR detection circuit with a quality factor

$$
Q_{\mathrm{LCR}}=100 \text { and } \tau_{\mathrm{LCR}}=T_{1} \text {. }
$$


$T$ value the data set representing the signal is reduced to a single point. If the signal is measured with a transient recorder, this reduction of the data is done after data acquisition [7]. One possibility is to calculate the amplitude of the spectral component at $\omega_{\mathrm{LCR}}$ of the signal caused by the detection pulse. Another option is to sum up the absolute values of all data points after a baseline correction. In the case of $T_{1} \ll T_{\mathrm{LCR}}$, it might be preferable to sum up only the data points during the first half-period of the oscillating signal, which is equivalent to the use of a boxcar averager for detection.

The effect of the mw detection pulse on the magnetization depends also on $T_{2}$. If $T_{2}$ is long enough that the nutation of the magnetization during the pulse lasts at least for one period $T_{\mathrm{LCR}}$, optimum sensitivity is obtained with a nutation frequency $\omega_{\mathrm{TN}}=\omega_{\mathrm{LCR}}$. If the decay of the magnetization can be refocused, the signal intensity can be further improved by periodically inverting the phase of the mw field. This results in a series of rotary echoes as in a TN-LOD EPR experiment [7]. For $T_{2} \ll 2 \pi / \omega_{\mathrm{TN}}$, the magnetization does not nutate, but a signal can still be observed because of the decay of $M_{z}$ during the pulse. It is therefore possible to measure $T_{1}$ for very short values of $T_{2}$.

For the two methods IR-LOD $T_{1}$ and SR-LOD $T_{1}$, there exists a lower but not an upper limit for $T_{1}$. This is mainly caused by two reasons. First, the resolution of the transient recorder is limited. For a reasonable fit of the data, a few data points should be collected until $M_{\mathrm{eq}}$ is reached. Second, because of the finite rise and fall time of the mw switches and the finite bandwidth $\Delta \omega$ of the $\mathrm{mw}$ resonator, the edges of the pulses can no longer be considered as infinitely short, whenever the switching times or $2 \pi / \Delta \omega$ are longer than $T_{1}$. In this case the decay of the observed signal is determined by both the relaxation time $T_{1}$ and the time constant of the fall time of the preparation pulse. This effect can be reduced by reducing the switching times and by lowering the quality factor of the resonator. With our setup, $T_{1}$ values down to approximately $20 \mathrm{~ns}$ can be measured.

A signal is not only induced in the coil by the change of $M_{z}$ during the detection pulse but also during the inversion or saturation pulse (Fig. lc). Since in most experiments the detection pulse is applied before the signal induced by the preparation pulse has decayed, the observed time trace during and after the detection pulse is a superposition of both signals. Consequently, when time $T$ is incremented in an IR-LOD $T_{1}$ or SR-LOD $T_{1}$ experiment, the relaxation curve is modulated with $\omega_{\mathrm{LCR}}$. Only in the case of $T_{1} \gg \tau_{\mathrm{LCR}}$, no additional procedures to reduce this unwanted modulation are required, because the shortest value of $T$ can be chosen large enough so that the signal induced in the LCR circuit by the preparation pulse has decayed at the time the detection pulse is applied.

\subsection{Removing the Unwanted Oscillations on the Relaxation Curve}

We describe three approaches to remove the unwanted oscillation on the relaxation curve: (a) subtraction of the data recorded in an experiment without detec- 
tion pulse from the original data, (b) calculation of the moving average over $T_{\mathrm{LCR}}$, (c) using an inversion sequence which consists of two pulses with the second pulse annihilating the signal induced by the first pulse. It is also possible to reduce $Q_{\mathrm{LCR}}$ to get a shorter $\tau_{\mathrm{LCR}}$ value, but this in turn reduces the sensitivity and is not a valuable alternative for short $T_{1}$ values.

\subsubsection{Subtracting the Blind Signal}

The signal induced by the inversion or saturation pulse can be removed by subtracting the signal obtained in an experiment without detection pulse (blind signal). To get meaningful results, two different mw channels should be used for the preparation and the detection pulse. The blind signal is obtained with a fully attenuated detection pulse; all other parameters remain the same. With this procedure not only the signal induced by the first pulse is subtracted but also spikes induced by other mw devices like the switches or the TWT amplifier are eliminated. The subtraction procedure is demonstrated in Fig. $2 a$ and $b$ for an SR experiment on natural dolomite. Figure $2 \mathrm{a}$ shows the original relaxation curve, while in Fig. $2 \mathrm{~b}$ the blind signals are subtracted separately for each trace. The corrected relaxation curve is still slightly modulated. Although recording a blind signal doubles the measuring time, we used this approach in all the experiments.

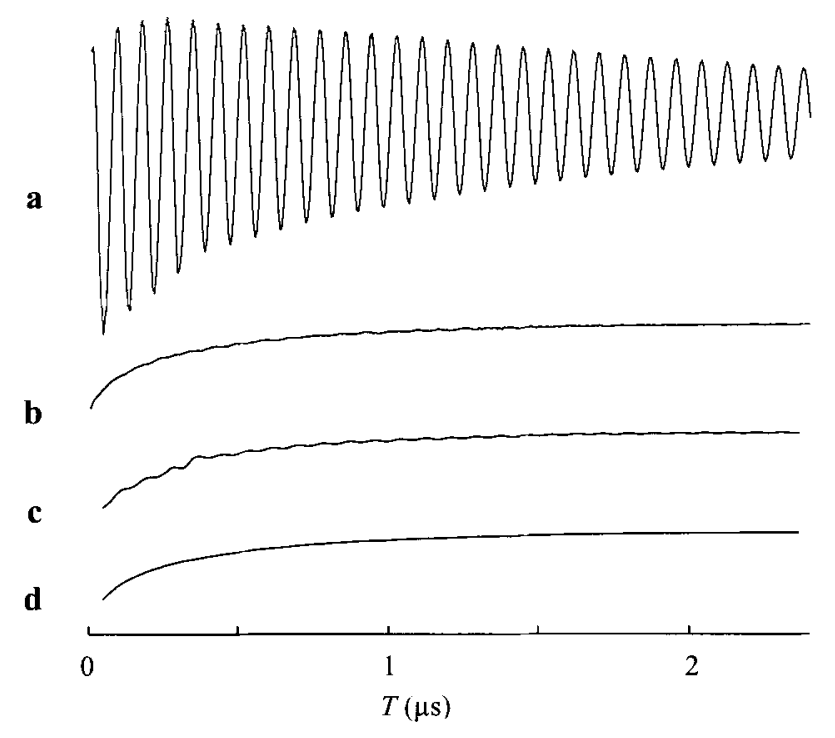

Fig. 2. SR-LOD $T_{1}$ experiment on $\mathrm{Mn}(\mathrm{II})$ centers in natural dolomite. a Initial relaxation curve after reduction of each transient trace observed at a fixed $T$ value to a single point. $\mathbf{b}$ With subtraction of blind signals. $c$ With a moving average over one modulation period of the detection circuit.

d With subtraction of the blind signals and moving average over one modulation period. 


\subsubsection{Calculating the Moving Average over the Period $T_{\mathrm{LCR}}$}

A modulation with frequency $\omega$ and constant amplitude can be removed by calculating the moving average over a period $T_{\mathrm{p}}=2 \pi / \omega$ or an integer multiple of it. Since the relaxation curve observed in the IR-LOD $T_{1}$ and SR-LOD $T_{1}$ experiments is often described by Eq. (1), superimposed by an exponentially damped cosine function with frequency $\omega_{\mathrm{LCR}}$ and time constant $\tau_{\mathrm{LCR}}$ originating from the preparation pulse, the moving average can be used to reduce the modulation. To show the efficiency of this approach, we discuss how the moving average influences the relaxation time $T_{1}$, what the residual is when the modulation is exponentially damped, and what the consequences are when $T_{\mathrm{p}}$ is not exactly a multiple of the difference between two adjacent data points.

First we integrate $M_{z}$ in Eq. (1) over a time period $\delta$ which is equivalent to a moving average over $\delta$ with an infinite number of points in this interval

$$
\begin{aligned}
\bar{M}_{z}(t) & =\frac{1}{\delta} \int_{t-\delta / 2}^{t+\delta / 2}\left[M_{\mathrm{eq}}+\left[M_{z}(0)-M_{\mathrm{eq}}\right] \exp \left(\frac{-t^{\prime}}{T_{1}}\right)\right] \mathrm{d} t^{\prime} \\
& =M_{\mathrm{eq}}+\frac{T_{1}}{\delta / 2} \sinh \left(\frac{\delta / 2}{T_{1}}\right)\left[M_{z}(0)-M_{\mathrm{eq}}\right] \exp \left(\frac{-t}{T_{1}}\right) .
\end{aligned}
$$

The result shows that for $\delta \ll T_{1}$ we get $\bar{M}_{z}(t) \approx M_{z}(t)$. If $\delta$ is in the order of $T_{1}, \bar{M}_{z}(t)$ has still the same shape as $M_{z}$, with the same relaxation time $T_{1}$ but with amplitude $T_{1} /(\delta / 2) \sinh \left[(\delta / 2) / T_{1}\right]>1$. This has consequences if the decay is multi-exponential. The relaxation curves with shorter $T_{1}$ values will become more intense relative to those with longer $T_{1}$ values.

For an exponentially damped cosine, $s(t)=\exp (-t / \tau) \cos (\omega t)$ with time constant $\tau$, integration over a modulation period $T_{\mathrm{p}}$ yields

$$
\begin{aligned}
\bar{s}(t) & =\frac{\omega}{2 \pi} \int_{t-\pi / \omega}^{t+\pi / \omega} \exp \left(\frac{-t^{\prime}}{\tau}\right) \cos \left(\omega t^{\prime}\right) \mathrm{d} t^{\prime} \\
& =\frac{\omega \tau \exp (-t / \tau)}{\pi\left(\mathrm{I}+\omega^{2} \tau^{2}\right)}[\omega \tau \sin (\omega t)-\cos (\omega t)] \sinh \left(\frac{\pi}{\omega \tau}\right) .
\end{aligned}
$$

In an LOD $T_{1}$ experiment with $\omega=\omega_{\mathrm{LCR}}, t=T$ and $\tau=\tau_{\mathrm{LCR}}=2 Q_{\mathrm{LCR}} / \omega_{\mathrm{LCR}}$ with $Q_{\mathrm{LCR}} \gg 1$, the ringing of the detection circuit after the preparation pulse, $s(T)=$ $\exp \left(-T / \tau_{\mathrm{LCR}}\right) \cos \left(\omega_{\mathrm{LCR}} T\right)$, is superimposed on the relaxation curve. The effect of the moving average can then be approximated by

$$
\bar{s}(T) \approx \frac{\exp \left(-T / \tau_{\mathrm{LCR}}\right) \sin \left(\omega_{\mathrm{LCR}} T\right)}{2 Q_{\mathrm{LCR}}} .
$$

This result shows that calculating a moving average over $T_{\mathrm{LCR}}$ reduces the modulation by a factor $2 Q_{\mathrm{LCR}}$ (Fig. 2c). But for short $T$ values, instrumental artifacts 
are still present. Therefore even when calculating a moving average over a modulation period, a blind signal should be subtracted. The result obtained after a moving average and subtraction of the blind signal is shown in Fig. 2d.

For an integration interval which deviates by $2 \Delta / \omega$ from $T_{p}$, we find

$$
\begin{aligned}
\bar{s}(t)= & \frac{\omega}{2(\pi+\Delta)} \int_{t-(\pi+\Delta) / \omega}^{t+(\pi+\Delta) / \omega} \exp \left(\frac{-t^{\prime}}{\tau}\right) \cos \left(\omega t^{\prime}\right) \mathrm{d} t^{\prime} \\
= & \frac{\omega \tau \exp (-t / \tau)}{2(\pi+\Delta)\left(1+\omega^{2} \tau^{2}\right)}\left[\exp \left(\frac{\pi+\Delta}{\omega \tau}\right)[\omega \tau \sin (\omega t-\Delta)-\cos (\omega t-\Delta)]\right. \\
& \left.-\exp \left(-\frac{\pi+\Delta}{\omega \tau}\right)[\omega \tau \sin (\omega t+\Delta)-\cos (\omega t+\Delta)]\right]
\end{aligned}
$$

With the assumptions $\Delta \ll \pi$ and $Q_{\mathrm{LCR}} \gg 1$, we obtain

$$
\bar{s}(T) \approx \exp \left(-\frac{T}{\tau_{\mathrm{LCR}}}\right)\left(\frac{1}{2 Q_{\mathrm{LCR}}} \cos \Delta \sin \left(\omega_{\mathrm{LCR}} T\right)-\frac{1}{\pi+\Delta} \sin \Delta \cos \left(\omega_{\mathrm{LCR}} T\right)\right) .
$$

There are two oscillatory terms in Eq. (6) which differ in phase by $90^{\circ}$. The sine function dominates if

$$
\frac{\Delta}{\pi}<\frac{1}{2 Q_{\mathrm{LCR}}}
$$

In this case, we find the same result as in Eq. (4), weighted by $\cos \Delta$. If the other term dominates, the amplitude of the unwanted modulation is

$$
\bar{s}(T) \approx \frac{\exp \left(-T / \tau_{\mathrm{LCR}}\right) \cos \left(\omega_{\mathrm{LCR}} T\right)}{1+\pi / \Delta},
$$

and the original modulation is reduced by a factor $(1+\pi / \Delta)$. For example, for a moving average over 11 data points separated by $8 \mathrm{~ns}$ and an oscillation period of $84 \mathrm{~ns}$, the error is $4 \mathrm{~ns}$, corresponding to $\pi / \Delta=21$. The modulation amplitude is reduced to about $4.5 \%$ of the initial value. If the remaining modulation is still too strong, the procedure can be repeated.

In all the calculations shown above, we assumed that the relaxation of $M_{z}$ is exponential. Otherwise the recovery curve would be distorted because only exponential functions are not affected in their character by a moving average.

\subsubsection{Two-Pulse Inversion Sequence}

The LOD signal induced in the coil by the preparation sequence can also be minimized by an inversion sequence which consists of two pulses where the 


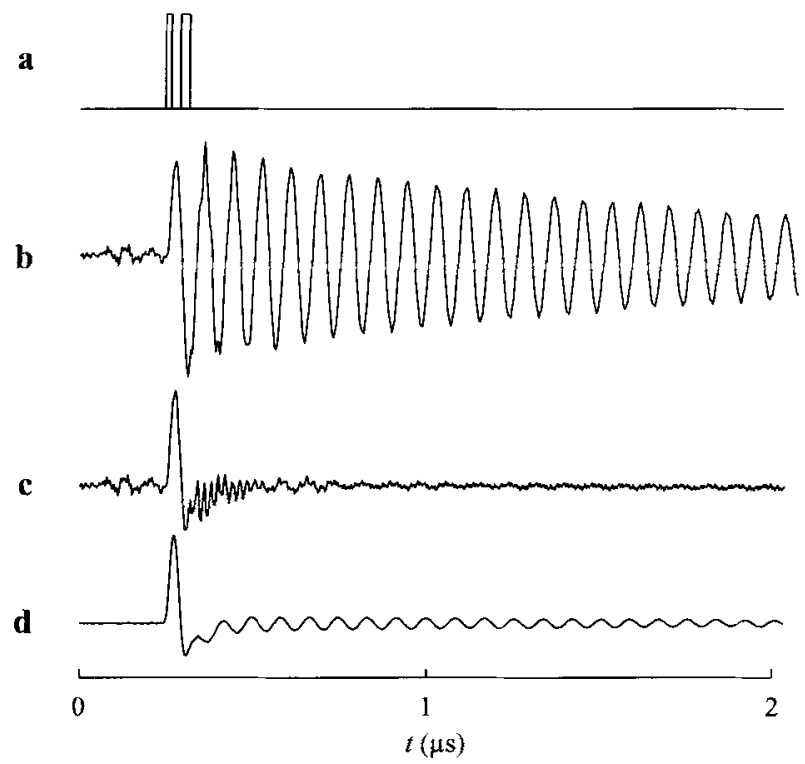

Fig. 3. Transient signal of DPPH observed during and after the two-pulse inversion sequence (without detection pulse). a Position and length of the pulses. b Only the first pulse was applied. c A second pulse is used to compensate the signal induced by the first pulse. d Inversion sequence with subtraction of the blind signal with fully damped mw power.

second pulse annihilates the signal induced by the first pulse (Fig. 3a). A rule of thumb for the optimum choice of length, intensity and interpulse delay of the two pulses cannot be given, because this strongly depends on the relaxation times. The easiest way to adjust these parameters is to observe the transient signal on the oscilloscope and to optimize the different parameters iteratively.

In Fig. $3 b$, only the first pulse is applied, resulting in a signal with a large amplitude. When the second pulse is optimized for minimum modulation at the end of the preparation sequence, the signal shown in Fig. $3 \mathrm{c}$ is obtained. The modulation of the signal after subtraction of the blind signal is stronger than the signal without this correction (Fig. 3d). This is because some of the artifacts which were reduced by the second pulse are added again by subtracting the blind signal. However, the high-frequency artifacts are strongly reduced, and the instrumental artifacts, which are clearly visible before the first pulse is applied, are completely removed.

\subsection{Direct Measurement of Longitudinal Relaxation}

An alternative way of measuring $T_{1}$ is to analyze the signal $\mathrm{d} M_{z}(t) / \mathrm{d} t$ which is induced after the preparation sequence by the relaxing $M_{z}$ magnetization. In analogy to the IR-LOD $T_{1}$ and SR-LOD $T_{1}$ experiments, an exponentially damped modulation with frequency $\omega_{\mathrm{LCR}}$ and time constant $\tau_{\mathrm{LCR}}$ originating from the ring- 
ing of the detection circuit is again superimposed on the relaxation curve. This time, only the instrumental artifacts can be removed by subtracting the blind signal which can be recorded either with a $B_{0}$ field far off-resonance or by reducing the mw power. To reduce the oscillations superimposed on the $\mathrm{d} M_{z}(t) / \mathrm{d} t$ relaxation curve, we have to calculate the moving average over $T_{\mathrm{LCR}}$ as explained in the previous section. As a consequence, this method is only feasible for an exponentially relaxing magnetization.

With this direct method, $T_{1}$ can be determined in a single experiment so that the recording time is strongly reduced compared to an IR-LOD $T_{1}$ or SR-LOD $T_{1}$ experiment. Furthermore, it is possible to carry out a 2-D field-swept EPR experiment with $T_{1}$ as a second dimension. However, this method is only appropriate for a small range of $T_{1}$ values. The lower limit is about $20 \mathrm{~ns}$ for the same reasons as discussed above for the IR-LOD $T_{1}$ and SR-LOD $T_{1}$ experiments. Since for this experiment the induced voltage is proportional to $\mathrm{d} M_{z}(t) / \mathrm{d} t$, the upper limit is in the order of $100 \mathrm{~ns}$ as the signal intensity scales with $1 / T_{1}$. This becomes evident by calculating the first derivative of Eq. (1). In addition, instrumental problems such as long-range baseline drifts or features which cannot be removed by subtracting a blind signal are responsible for this small time interval.

An alternative to the calculation of the moving average is to perform the experiment with a resonant circuit with a very low $Q_{\mathrm{LCR}}$ value so that after excitation the ringing of the detection circuit decays rapidly ( $\mathrm{P}$. Höfer, pers. commun.).

\section{Instrumentation}

The instrumental setup has been described in detail in ref. 7. All the experiments presented here were performed on a Bruker X-band pulse EPR spectrometer with an ESP-380E pulse bridge and an ELEXSYS E580 pulse controller and signal processing unit. We used a Bruker X-band ENDOR probehead (EN 4118X), rotated about the sample axis by $90^{\circ}$ so that the axis of the radio-frequency coil with inductance $L \approx 2.6 \mu \mathrm{H}$, acting as a pickup coil, was oriented parallel to the $\mathbf{B}_{0}$ field. One of the two semirigid cables from the coil to the ENDOR plugs at the outside of the probehead was short-circuited with the ground and acted as an inductance with a much smaller value than the inductance of the coil. The second semirigid cable was left open and operated as a capacitance to the ground with $C_{\mathrm{C}} \approx 50 \mathrm{pF}$. This already formed an LCR circuit with $\omega_{\mathrm{LCR}} / 2 \pi \approx 13 \mathrm{MHz}$ and a quality factor at room temperature of $Q_{\mathrm{LCR}}>100$. The resonant frequency could be adjusted within a few megacycles by using a tunable air capacitor with $C_{\mathrm{T}}=5-15 \mathrm{pF}$ in parallel to the capacitively coupled cable. The output voltage was detected with high impedance. The matching to the input impedance of the radio-frequency preamplifier (Avtech AV-141C) was done actively with a voltage follower (home-built). After preamplification, the signal was fed to the transient recorder of the spectrometer (SpecJet), where the transient signals could be added with a high repetition rate. 
This setup is much more sensitive than earlier designs because of the high filling factor of the detection coil, the high $Q_{\mathrm{LCR}}$ value, and the high resonant frequency $\omega_{\mathrm{LCR}}$ [7] which is kept constant throughout the whole experiment.

\section{Results and Discussion}

A sample of $\gamma$-irradiated herasil glass (100 kGy) was measured with the IR-LOD $T_{1}$ pulse sequence at $298 \mathrm{~K}$. To enhance signal intensity, the magnetization was two times refocused during the detection pulse of length $2.1 \mu$ s by switching the mw phase by $180^{\circ}$ after 420 and 1260 ns. The result is shown in Fig. 4 together with the residues between the observed and the fitted signal. A spinlattice relaxation time of $T_{1}=219 \mu \mathrm{s}$ was found. The same relaxation time was obtained with a standard IR experiment and two-pulse echo detection. The fit is slightly better by assuming a distribution of $T_{1}$ values (Fig. 4c) instead of a monoexponential decay (Fig. 4b). This is reasonable because the strong $\gamma$-irradiation leads to a high concentration of defect centers. Since this method of generating defects is not very specific, fluctuations of local defect concentrations may occur. In addition, in a glass the environment of the centers is not very well defined.

As a second example, the SR-LOD $T_{1}$ method was used to study a natural dolomite at $250 \mathrm{~K}$ where two overlapping spectra with different longitudinal relaxation behavior originating from $\mathrm{Mn}$ (II) that substitutes $\mathrm{Ca}(\mathrm{II})$ and $\mathrm{Mg}(\mathrm{II})$ can be observed (J. Granwehr et al., unpubl.). The relaxation curve shown in Fig. 5
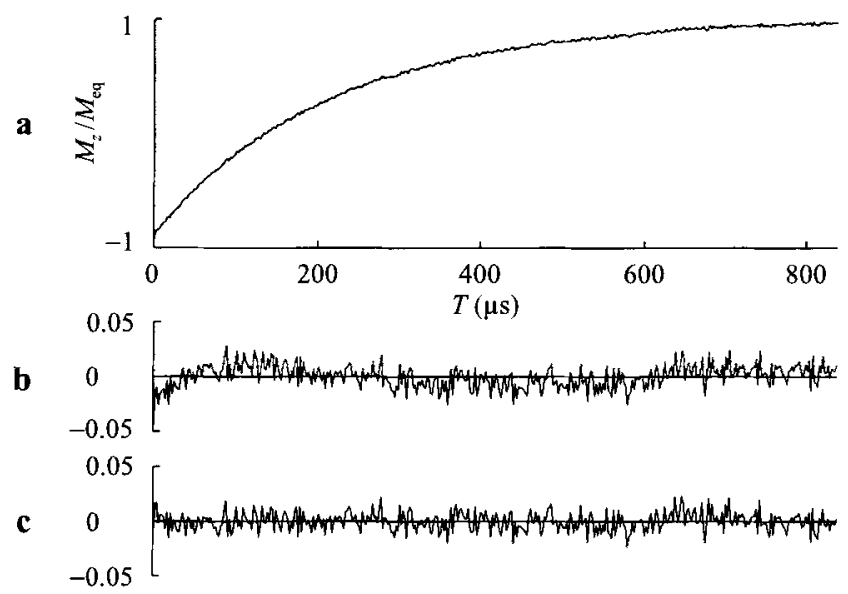

Fig. 4. IR-LOD $T_{1}$ measurement of $\gamma$-irradiated herasil glass at $298 \mathrm{~K}, B_{0}=343.2 \mathrm{mT}$ and $v_{\mathrm{mw}}=$ $9.614 \mathrm{GHz}$. The length of the inversion pulse was $t_{\pi}=22 \mathrm{~ns}$, the length of the detection pulse was $2.1 \mu \mathrm{s}$ with two phase changes. The nutation frequency was adjusted to $\omega_{\mathrm{CR}} .400$ data points were collected. The acquisition time was 15 min, including the measurement of the blind signal. a Experimental relaxation curve. b Residuals between the experimental relaxation curve shown in a, and the fitted curve assuming an exponential recovery of $M_{z}$. $\mathbf{c}$ Residuals between the experimental relaxation curve and the fitted curve, assuming a distribution of $T_{1}$ values. 


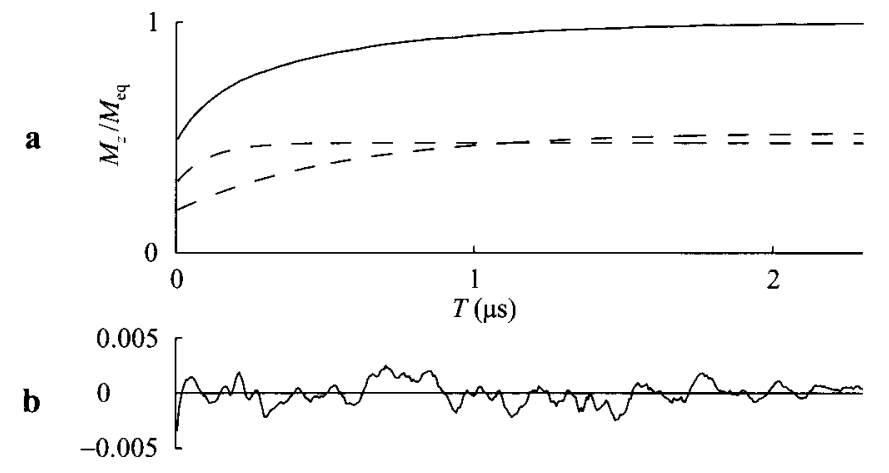

Fig. 5. SR-LOD $T_{1}$ measurement on $\mathrm{Mn}(\mathrm{II})$ centers in natural dolomite at $250 \mathrm{~K}, B_{0}=352.2 \mathrm{mT}$ and $v_{\mathrm{mw}}=9.622 \mathrm{GHz}$. The length of the saturation pulse was $420 \mathrm{~ns}$, the length of the detection pulse was $44 \mathrm{~ns}$. The mw power was $500 \mathrm{~W}$ for the saturation pulse and $150 \mathrm{~W}$ for the detection pulse. 400 data points were collected. The acquisition time was $15 \mathrm{~min}$, including the measurement of the blind signal. a Solid line: experimental relaxation curve, smoothed with a moving average. Dashed lines: components of the relaxation curve obtained by the fitting of the experimental data.

b Residuals between the experimental curve and the sum of the fitted curves.

was obtained by calculating the moving average over $T_{\mathrm{LCR}}$. The longitudinal relaxation times are found to be $T_{1}(1)=110 \mathrm{~ns}$ and $T_{1}(2)=568 \mathrm{~ns}$.

As a last example, we determined the relaxation time $T_{1}$ of a powder of DPPH with the SR-LOD $T_{1}$ method, as well as the direct measurement of $T_{1}$. After recording the SR-LOD $T_{1}$ data consisting of 100 data points with a transient recorder, three different procedures for data analysis have been used (Fig. 6). (a) In Fig. 6a, the amplitude of the spectral component at frequency $\omega_{\mathrm{LCR}}$ of the signal for each $T$ value after the detection pulse was determined, as in the previous examples. (b) A second approach for data analysis, shown in Fig. 6b, uses the sum of the two data points with maximum signal during the detection pulse. With the two approaches $\mathrm{a}$ and $\mathrm{b}$ for data analysis, time $T$ between inversion and detection pulse was incremented step by step, leading to the SR-LOD $T_{1}$ relaxation curve. (c) In the third scheme, the same data set was used for a different analysis. Since $M_{z}$ has fully relaxed for $T>400 \mathrm{~ns}$ (see Fig. 6a), the signal induced by the detection pulse does not depend on the preparation pulse anymore. Therefore for any of these traces the same result is expected in a direct measurement of $T_{1}$ when only the signal after the detection pulse is considered. To increase the signal-to-noise ratio, the 50 traces with $T>500$ ns were added before calculating two consecutive moving averages over $T_{\mathrm{LCR}}$. The result is shown in Fig. 6c. The relaxation times found with these three methods for data analysis are (a) $T_{1}=59.2 \mathrm{~ns}$, (b) $T_{1}=56.7 \mathrm{~ns}$, and (c) $T_{1}=57.9 \mathrm{~ns}$.

It should be noted that the first two methods which represent just two different ways of analyzing the data provide basically the same information. The third method uses a different mechanism for measuring $T_{1}$ and can therefore be used to verify the results obtained with the first two schemes. 

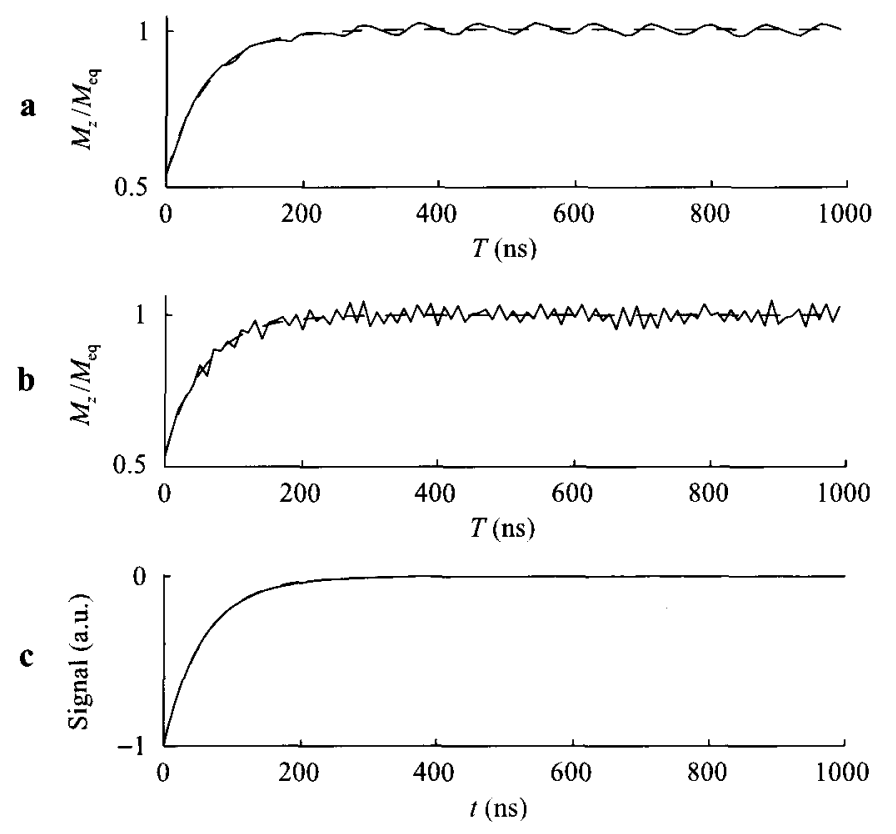

Fig. 6. SR-LOD $T_{1}$ measurement of a powder of DPPH at $298 \mathrm{~K}, B_{0}=343.3 \mathrm{mT}$ and $v_{\mathrm{mw}}=9.630 \mathrm{GHz}$. The length of the saturation pulse was $420 \mathrm{~ns}$, the length of the detection pulse was $84 \mathrm{~ns}$. The mw power was $500 \mathrm{~W}$ for both pulses. In the $T$ dimension 100 points were recorded, separated by $10 \mathrm{~ns}$. The acquisition time was $4 \mathrm{~min}$, including the measurement of the blind signal. The solid lines represent the experimental relaxation curves, the dashed lines are the corresponding fits. a From each trace in the $t$ dimension the spectral component at $\omega_{\text {ICR }}$ was calculated. b Only the two data points with maximum signal during the detection pulse were used. c Signal induced directly by the recovery of $M_{z}$ after the detection pulse, smoothed with two moving averages.

The same analysis of the relaxation curve after an mw excitation can be performed with the data from the example with the preparation sequence consisting of two pulses (Fig. 3d). In this case, it is sufficient to use a single moving average over $T_{\mathrm{LCR}}$ (curve not shown). A value of $T_{1}=57.8 \mathrm{~ns}$ has been found in this experiment.

The relaxation times of DPPH have extensively been investigated in the literature. Since $T_{2}$ is between $10^{-8}$ and $10^{-7} \mathrm{~s}$ and very close to the $T_{1}$ value, other recording techniques perform equally well. In ref. 10 , for example, a value of $T_{1}=56$ ns was reported.

\section{Conclusions}

Longitudinal detection represents an alternative way to measure spin-lattice relaxation times with IR and SR experiments. The approach overcomes most of the limitations inherent in conventional detection techniques. Echo formation is not required, and the method is free of the instrumental deadtime; high-power 
mw pulses can be applied even during detection. As a consequence, a very broad range of $T_{1}$ values down to about $20 \mathrm{~ns}$, independent of $T_{2}$ or the ratio $T_{1} / T_{2}$, can be measured with the same method, and the data which represent the recovery of $M_{z}$ are easy to analyze.

A blind signal recorded with a minimum of mw power for the detection pulse has to be subtracted to cancel the ringing of the detection circuit originating from the preparation pulse and to reduce instrumental artifacts. Another possibility to reduce the ringing is to split the inversion pulse into two pulses, where the signal induced in the detection circuit by the second pulse compensates for the signal induced by the first pulse. However, in this case the various parameters have to be adjusted carefully, and an additional evolution time between the two pulses is introduced which may influence the result. With these techniques any relaxation process can be investigated. If relaxation is described as an exponential or a sum of exponentials, unwanted signals can be reduced by calculating a moving average over a period of the resonant frequency of the detection circuit.

The experiments which have been carried out on a commercial spectrometer with a commercial ENDOR probehead require a minimum of additional equipment, are easy to implement and can be performed routinely.

We described two schemes for data acquisition. In the first scheme the whole trace of the signal after the detection pulse is recorded. Each trace is then reduced by an appropriate method to a single point in the relaxation curve. This approach has the advantage that by filtering, the desired information can be optimized after data acquisition, and in some cases the data can be analyzed in different ways to verify the results. Another way of data acquisition is to integrate part of the signal with a boxcar. With this method it is not possible to selectively filter individual frequency components of the signal. But the blind signal can directly be subtracted with a similar experimental procedure as is used for phase cycling, namely by altering the intensity of the detection pulse between two experiments instead of changing the phase. This is not possible on most spectrometers when a transient signal is recorded.

We have also demonstrated that $T_{1}$ can be determined by directly observing the signal induced by the time-dependent magnetization during a relaxation process. In this case, the first derivative of the relaxation curve is recorded, in contrast to the IR and SR methods where the relaxation of $M_{z}$ is observed. The experiment is easy to perform, and one gets the whole relaxation curve in a single shot which reduces the measuring time considerably, even if several time traces have to be accumulated. Since the method is fundamentally different from the standard IR or SR experiments, it provides an additional means to verify the results, in particular because the information can be extracted from the same set of data. The applicability of the direct method is restricted to exponential relaxation processes with $T_{1}$ values between 20 and approximately $100 \mathrm{~ns}$, which makes the technique an alternative only for a limited number of samples. 


\section{Acknowledgements}

We thank Jörg Forrer for his technical support and Dr. Andreas Gehring for the dolomite sample. Furthermore we are grateful to Dr. Peter Höfer from Bruker Analytik $\mathrm{GmbH}$ for interesting discussions and for performing the experiment with the direct determination of $T_{1}$ with a similar setup. This work has been supported by the Swiss National Science Foundation.

\section{References}

1. Beck W.F., Innes J.B., Lynch J.B., Brudvig G.W.: J. Magn. Reson. 91, 12 (1991)

2. Hyde J.S. in: Time-Domain Electron Spin Resonance (Kevan L., Schwartz R.N., eds.), p. 1. New York: Wiley 1979.

3. Bloembergen N., Damon R.W.: Phys. Rev. 85, 699 (1952)

4. Strutz T., Witowski A.M., de Bekker R.E.M., Wyder P.: Appl. Phys. Lett. 57, 831 (1990)

5. Pescia J.: Ann. Phys. 10, 389 (1965)

6. Schweiger A., Ernst R.R.: J. Magn. Reson. 77, 512 (1988)

7. Granwehr J., Forrer J., Schweiger A.: J. Magn. Reson., in press.

8. Ernst R.R., Bodenhausen G., Wokaun A.: Principles of Nuclear Magnetic Resonance in One and Two Dimensions. Oxford: Clarendon Press 1987.

9. Hoffmann E., Schweiger A.: Appl. Magn. Reson. 9, 1 (1995)

10. Brändle R., Krüger G.J., Müller-Warmuth W.: Z. Naturforsch. 25, 1 (1970)

Authors' address: Arthur Schweiger, Physical Chemistry Laboratory, ETH Zentrum, CH-8092 Zurich, Switzerland 